\title{
CONTROLADOR DE PITCH HÍBRIDO OPTIMIZADO CON ALGORITMO GENÉTICO PARA AEROGENERADORES FLOTANTES
}

\author{
Carlos L. Serrano-Barreto ${ }^{10000-0003-1228-3135]}$ \\ Facultad de Informática, Universidad Complutense de Madrid, 28040 Madrid, España, carser06@ucm.es \\ Matilde Santos $3[0000-0003-1993-8368]$ \\ Instituto de Tecnología del Conocimiento, Universidad Complutense de Madrid, España, msantos@ucm.es \\ Jesús Enrique Sierra-García ${ }^{2[0000-0001-6088-9954] ~}$ \\ Universidad de Burgos, Burgos, España, jesierra@ubu.es
}

\begin{abstract}
Resumen
El control de las turbinas eólicas flotantes (Floating Offshore Wind Turbine, FOWT) es complejo debido al tamaño de estas turbinas, las fuertes cargas a las que están sometidas, y su dinámica fuertemente no lineal. Además, la acción de control de la turbina puede influir en la estabilidad del dispositivo flotante. En este trabajo se diseña un controlador híbrido inteligente para el control del ángulo de cabeceo de las palas de un aerogenerador flotante. El objetivo del control es reducir la vibración del dispositivo flotante sin comprometer la potencia de salida. En concreto, se desarrolla un controlador borroso inteligente y una implementación difusa de la relación velocidad del viento-ángulo de cabeceo. Esta estrategia inteligente de control es validada con la referencia proporcionada por el software de simulación de aerogeneradores FAST, que implementa un controlador PI de ganancia programada. Además, se analizan los efectos de estas diferentes estrategias de control sobre las vibraciones en la estructura.
\end{abstract}

Palabras clave: Control híbrido inteligente, Control de palas, Control borroso, Vibraciones, Turbina eólica flotante, Energía eólica.

\section{INTRODUCCIÓN}

Debido a la creciente demanda de energía y al aumento de la contaminación por residuos de carbono, se buscan formas alternativas de producir energía limpia y renovable $[1,2]$. Entre ellas, la energía eólica ha demostrado ser muy eficiente. La energía eólica incluye aerogeneradores terrestres y marinos. Los primeros, aunque son una tecnología madura, tienen hoy en día limitaciones en cuanto a espacio disponible y daños ambientales. Por ello, las turbinas eólicas marinas, principalmente las flotantes, están surgiendo como una prometedora solución en materia de energías renovables [3-5].
Pero el control de las turbinas eólicas flotantes (Floating Offshore Wind Turbine, FOWT) es complejo debido al gran tamaño de estas turbinas, las fuertes cargas (olas, viento, corrientes, ...) a las que están sometidas, y su dinámica fuertemente no lineal [6]. Además, la acción de control de la turbina, principalmente los cambios del ángulo de las palas, puede influir en la estabilidad del dispositivo flotante, comprometiendo la eficiencia y la integridad de la propia turbina [7-9].

En este trabajo se propone la utilización de un controlador híbrido inteligente para el ángulo de las palas de una FOWT, con el objetivo de reducir la vibración de la estructura flotante sin comprometer la potencia eólica de salida. Diferentes técnicas avanzadas e inteligentes han demostrado ser eficientes para estos sistemas complejos [11, 12]. En particular, en este trabajo se desarrolla un controlador difuso inteligente y una implementación difusa de la relación velocidad del viento-ángulo de inclinación. Los parámetros del controlador se han ajustado mediante un algoritmo genético. El controlador ha sido validado con la referencia proporcionada por el software de simulación de aerogeneradores FAST, que implementa un controlador PI de ganancia programada (GSPI). Además, se comparan los efectos de estas diferentes estrategias de control sobre las vibraciones de la estructura flotante.

La estructura del artículo es la siguiente. La sección 2 describe el control del ángulo de cabeceo de las palas de una turbina flotante. La sección 3 presenta la nueva estrategia de control híbrido. En la sección 4 se muestran y discuten los resultados. El artículo finaliza con las conclusiones y el trabajo futuro.

\section{CONTROL DE CABECEO DE UNA TURBINA FLOTANTE}

El viento y la potencia que se puede extraer de un aerogenerador vienen dados por la siguiente relación cúbica [4]: 


$$
P=0.5 \rho A v^{3}
$$

Donde $P$ es la potencia $(W), \rho$ es la densidad del aire $\left(\mathrm{Kg} / \mathrm{m}^{3}\right), A$ es el área barrida por las palas $\left(\mathrm{m}^{2}\right)$ y $v$ es la velocidad del viento $(\mathrm{m} / \mathrm{s})$.

Según el límite de Betz [11], la energía eólica máxima que puede convertirse en energía eléctrica es del 59\%. La energía que puede generar el aerogenerador depende del coeficiente de potencia $C p$, que es específico para cada tipo de turbina. El coeficiente $C p$ es una función de la relación de velocidad de las palas, $\lambda, \mathrm{y}$ del ángulo de inclinación de las palas, $\beta$.

$$
P_{\omega t}=0.5 \rho A v^{3} C p(\beta, \lambda)
$$

La aproximación del coeficiente $C p$ utilizada en las simulaciones viene dada por [11]:

$$
C p=0.5176\left(\frac{116}{\lambda i}-0.4 \beta-5\right) e^{-\frac{21}{\lambda i}}+0.0068 \lambda
$$

Donde la variable $\lambda_{i}$ no tiene significado físico y se puede obtener como,

$$
\frac{1}{\lambda i}=\frac{1}{\lambda+0.08 \beta}-\frac{0.035}{3 \beta+1}
$$

La relación entre el cambio de velocidad punta del rotor y la velocidad del viento es:

$$
\lambda=\frac{w \cdot R}{v}
$$

Donde $w(\mathrm{rad} / \mathrm{s})$ es la velocidad angular del rotor y $R$ (m) el radio efectivo de las palas.

Existen cuatro regiones de funcionamiento en función de la velocidad del viento, a saber:

\section{POTENCIA EXTRAIDA (kW) / VELOCIDAD VIENTO $(\mathrm{m} / \mathrm{s})$}

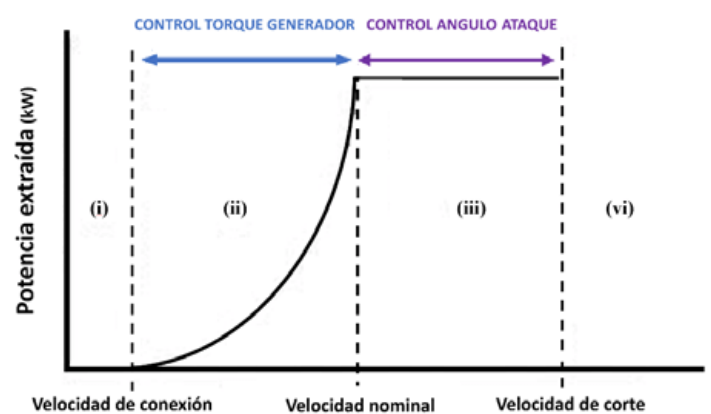

Figura 1: Regiones de funcionamiento de la turbina en función del viento[12].

Para mantener la potencia de salida en la región (iii) se requiere un sistema de control que ajuste el ángulo de paso de las palas (pitch) [13].
Se ha simulado el aerogenerador flotante NREL de $5 \mathrm{MW}$, tipo barcaza (con plataforma ITI). Las principales características de esta turbina en relación al control son: velocidad nominal del viento, $12,1 \mathrm{~m} / \mathrm{s}$; longitud de las palas, 61,609 m; velocidad de corte del viento, $25 \mathrm{~m} / \mathrm{s}$, velocidad nominal del rotor, $w_{\text {ref }} 12,1$ rpm [14].

\section{METODOLOGÍA}

En este trabajo utilizaremos como referencia para contrastar los resultados el control PI con ganancia programada (GSPI) que el software de simulación FAST tiene implementado para este aerogenerador.

Las variaciones de la velocidad del viento provocan cambios continuos en el punto de funcionamiento del aerogenerador, lo que a su vez provoca un cambio en el control. Los controladores borrosos permiten hacer frente a las no linealidades del sistema y superar las variaciones de la velocidad del viento y las turbulencias, proporcionando un modelo que relaciona cada velocidad del viento a la entrada [15]. Mediante la utilización de algoritmos genéticos (AG) se optimiza el punto de operación alrededor del cual trabajará el controlador de la turbina eólica.

La estructura de esta estrategia de control inteligente se muestra en la Figura 2. Se propone la implementación de un sistema de lógica híbrida borrosa porque las turbinas flotantes son sistemas fuertemente no lineales, y este tipo de controlador produce una respuesta más suave. La velocidad del rotor se controla utilizando el ángulo de cabeceo de las palas con el objetivo de obtener la potencia nominal de la turbina.

La entrada del controlador PD borroso incremental es la velocidad actual del rotor, y la referencia es la velocidad del rotor deseada para producir la potencia nominal.

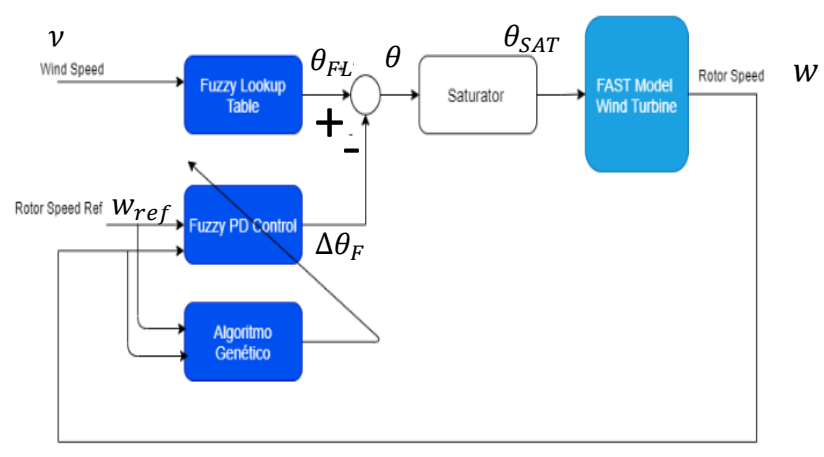

Figura 2: Controlador inteligente híbrido con estrategia de tabla de búsqueda difusa.

Se calcula el error como la diferencia entre estas dos señales para así poder aumentar o disminuir el ángulo 
de cabeceo de la turbina en función de la región de funcionamiento del aerogenerador. El saturador limita la señal de control a $25^{\circ}$, que es el ángulo de cabeceo máximo para estas turbinas [16].

El controlador borroso es un PD incremental tipo Takagi-Sugeno (equivalente a un regulador PI). Tiene como entradas el error de velocidad del rotor, $\omega_{e}$ (rpm) (7) y su derivada, $\dot{\omega}_{e}(\mathrm{rpm} / \mathrm{s})$.

$$
w_{e}=w_{\text {ref }}-w
$$

La referencia $\omega_{\text {ref }}$ se ha fijado en 12,1 rpm. Para las dos variables de entrada se asignaron tres conjuntos difusos triangulares y trapezoidales en los extremos: Positivo, Negativo y Cero. El error y la derivada del error se han normalizado a $[-1,1]$.

Las reglas del controlador difuso Takagi-Sugeno se han expresado de la siguiente forma,

$$
\text { Rule(i): if } w_{e} \text { is } A_{1 i} \text { and } \dot{w}_{e} \text { is } A_{2 n} \text { then } y_{i}
$$

Donde $A_{1 i}$ y $A_{2 i}$ representan los conjuntos difusos de las entradas $w_{e} \mathrm{y} \dot{w}_{e}$, y la variable $y_{i}$ representa la salida del controlador. La variable de salida es el incremento del ángulo de paso, $\Delta \theta_{F}\left({ }^{\circ}\right)$. Estas reglas se enumeran en la Tabla 1.

Tabla 1: Reglas del controlador difuso.

1. If $w_{e}$ is negative and $\dot{w}_{e}$ is negative, then $\Delta \theta_{F}$ is -5 .

2. If $w_{e}$ is negative and $\dot{w}_{e}$ is zero, then $\Delta \theta_{F}$ is -2.5 .

3. If $w_{e}$ is negative and $\dot{w}_{e}$ is positive, then $\Delta \theta_{F}$ is 0 .

4. If $w_{e}$ is zero and $\dot{w}_{e}$ is negative, then $\Delta \theta_{F}$ is -2.5 .

5. If $w_{e}$ is zero and $\dot{w}_{e}$ is zero, then $\Delta \theta_{F}$ is 0 .

6. If $w_{e}$ is zero and $\dot{w}_{e}$ is positive, then $\Delta \theta_{F}$ is 2.5 .

7. If $w_{e}$ is positive and $\dot{w}_{e}$ is negative, then $\Delta \theta_{F}$ is 0 .

8. If $w_{e}$ is positive and $\dot{w}_{e}$ is zero, then $\Delta \theta_{F}$ is 2.5 .

9. If $w_{e}$ is positive and $\dot{w}_{e}$ is positive, then $\Delta \theta_{F}$ is 5 .

El ángulo de cabeceo que se envía al modelo de la FOWT en FAST se obtiene con la siguiente relación:

$$
\theta=\theta_{F L}-\Delta \theta_{F}
$$

Por otro lado, la tabla de búsqueda se ha implementado utilizando también lógica difusa. La entrada es el viento y la salida es la referencia del ángulo de paso. Esta referencia es corregida por el controlador incremental borroso-PD.

La tabla de búsqueda difusa tiene como entrada la velocidad del viento, representada por 3 conjuntos borrosos: Positivo, Negativo y Cero, utilizados para crear la función de mapeo donde las reglas son la relación entre cada entrada y la salida. La salida es la referencia del ángulo de cabeceo. La función de pertenencia difusa y las reglas difusas del sistema están inspiradas en [15].

El algoritmo genético se utilizar para minimizar el error entre la velocidad de referencia del rotor y la velocidad actual del rotor. Con él se sintonizan los coeficientes del controlador borroso, GE (Ganancia del error) y GDE (Ganancia de la derivada del error).

El algoritmo genético tiene la siguiente configuración. La población inicial es de 50 individuos, con dos cromosomas cada uno, GE y GDE; la probabilidad de cruce es 0.8 ; la probabilidad de mutación es 0.05 ; por último, la función de coste es:

$$
J=\sum \frac{\left|\left(\omega_{\text {ref }}-\omega\right)\right|}{n}
$$

Donde $n$ es el número de muestras obtenidas en la simulación.

Por último, la condición de parada del algoritmo se establece cuando la función de coste es menor a uno, ya que un error de una revolución por minuto es aceptable en el control de velocidad del rotor. El algoritmo se ejecuta off-line para ajustar los parámetros del controlador.

\section{RESULTADOS DE SIMULACIÓN Y DISCUSIÓN}

Para la simulación del aerogenerador se utilizó el software NREL FAST v8 [17] y el controlador se implementó en Matlab/Simulink. El enfoque de control propuesto se compara con el controlador PI de ganancia programada (GSPI) del sistema FAST [17].

El perfil del viento es una señal aleatoria generada por la herramienta TurbSim de NREL [17]. La velocidad media del viento es de $17 \mathrm{~m} / \mathrm{s}$, con un valor mínimo de $10,3 \mathrm{~m} / \mathrm{s}$ y un máximo de $23,6 \mathrm{~m} / \mathrm{s}$

El ángulo de cabeceo obtenido en la simulación por ambos controladores se muestra en la Figura 3.

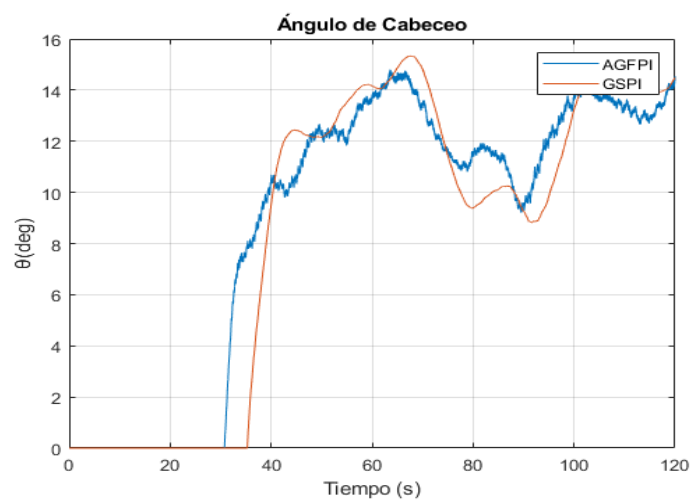

Figura 3: Ángulo de cabeceo. 
Se puede observar algo de ruido de alta frecuencia en el controlador borroso. Sin embargo, debido a la gran masa de la turbina eólica, ésta no se ve afectada por el mismo. Por otro lado, si se quisiera reducir el ruido de alta frecuencia bastaría con poner un filtro pasa bajo a la salida del sistema de control borroso.

La velocidad del rotor obtenida con cada controlador se muestra en la Figura 4. Ambos controladores son capaces de mantener la velocidad del rotor en la región (iii) de funcionamiento.

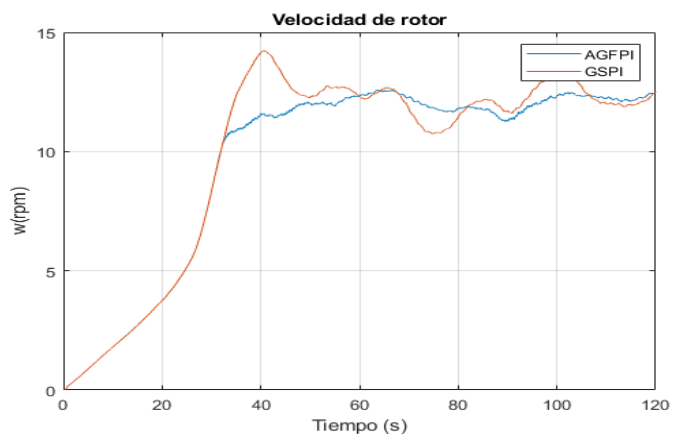

Figura 4: Velocidad de rotor.

Sin embargo, también se puede observar que la respuesta más estable la obtienen el controlador borroso (línea azul).

Por otro lado, la potencia generada durante la simulación debe ser constante, en torno a la potencia nominal, es decir, 5MW (Figura 5). En este caso también se obtienen resultados más estables con el controlador borroso.

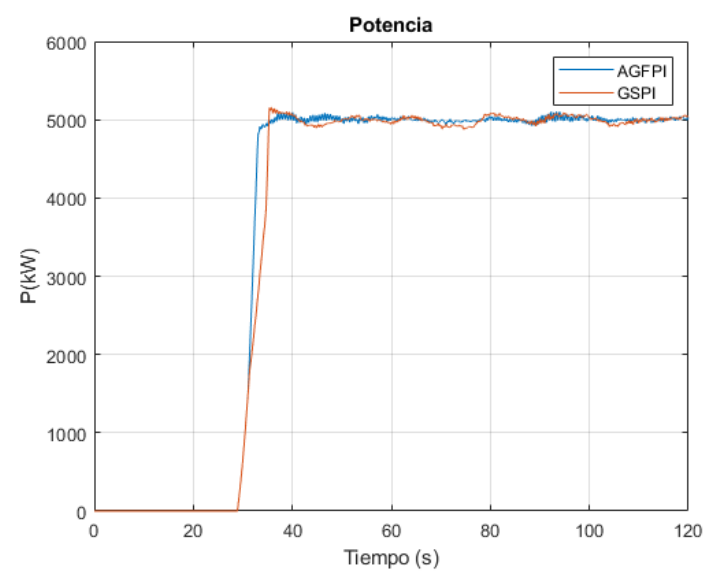

Figura 5: Potencia de salida.

Además, se han analizado los efectos de cada acción de control sobre la estabilidad de la turbina flotante. Para ello se utilizan dos variables: el cabeceo de la plataforma y el desplazamiento de la parte superior de la torre en la dirección proa-popa (TTDspFA). La primera indica el ángulo $\left(^{\circ}\right)$ de inclinación de la plataforma respecto a la horizontal, mientras que la segunda representa el desplazamiento $(\mathrm{m})$ de la parte superior de la torre respecto a la posición de equilibrio de la turbina. Estas mediciones se representan en las Figuras 6 y 7 para los diferentes enfoques de control.

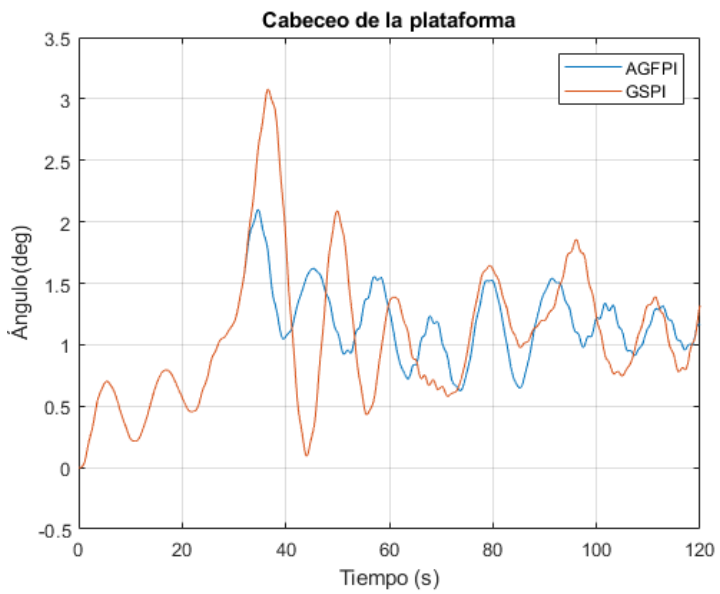

Figura 6: Cabeceo de la plataforma.

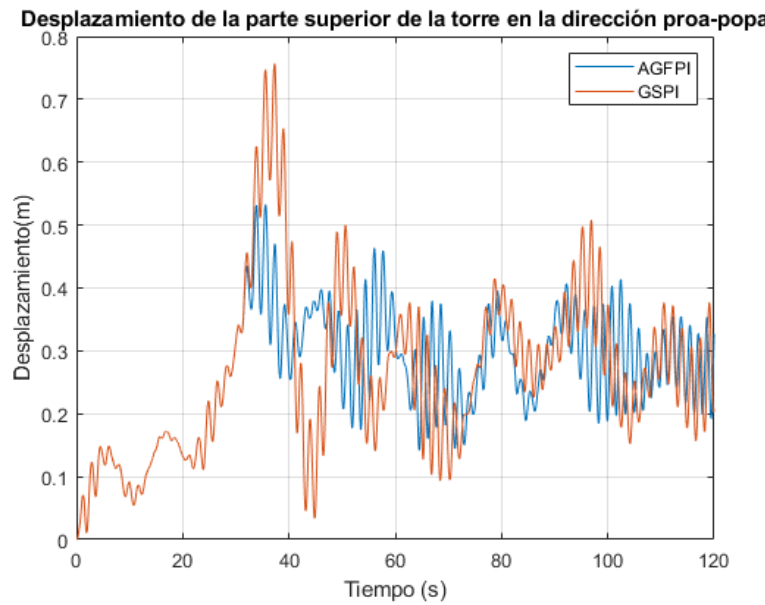

Figura 7: Desplazamiento de la parte superior de la torre en la dirección proa-popa.

Como se puede ver en esas figuras, el controlador GSPI genera mayores oscilaciones durante más tiempo. Por el contrario, el controlador borroso reduce las vibraciones y da mejor respuesta en el estado estacionario.

Es importante observar que ambos controladores se comportan igual hasta el segundo 32, tanto en la plataforma como en la torre. Esto se debe a que hasta ese momento reciben la misma señal de control, ya que el ángulo de cabeceo es cero para que el rotor se acelere lo más rápido posible.

Para evaluar el rendimiento de los controladores, además de la potencia de salida, se han analizado las vibraciones producidas en el sistema mediante la tasa de supresión de vibraciones, $\eta$ (10) [18], y el error cuadrático medio (RMSE) (11), 


$$
\begin{gathered}
\eta=\frac{\sigma_{G S F A T S}-\sigma_{\text {controller }}}{\sigma_{G S F A S T}} .100 \% \\
R M S E=\frac{\sigma}{\sqrt{n}}
\end{gathered}
$$

Donde $\sigma$ representa la desviación estándar de las oscilaciones en la turbina, tanto de la plataforma como de la torre, y $n$ es el número de muestras. La tasa de supresión se calcula en ambos casos con respecto a la referencia del controlador FAST, $\sigma_{G S F A S T}$.

Los resultados se muestran en la Tabla 2 y la Tabla 3.

Tabla 2: Rendimiento de los controladores con respecto al cabeceo de la plataforma

\begin{tabular}{lll}
\hline Controlador & $\begin{array}{l}\text { RMSE } \\
\text { normalizado }\end{array}$ & $\begin{array}{l}\text { Tasa de } \\
\text { supresión }(\%)\end{array}$ \\
\hline FAST & 1 & - \\
\hline Hybrid & 0.6754 & 32.4615 \\
\hline
\end{tabular}

Tabla 3: Rendimiento de los controladores con respecto al desplazamiento de la parte superior de la torre (TTDspFA)

\begin{tabular}{lll}
\hline Controlador & $\begin{array}{l}\text { RMSE } \\
\text { normalizado }\end{array}$ & $\begin{array}{l}\text { Tasa de } \\
\text { supresión }(\%)\end{array}$ \\
\hline FAST & 1 & - \\
\hline Hybrid & 0.7461 & 25.3888 \\
\hline
\end{tabular}

Se puede observar como el controlador borroso obtiene una mejora relevante en la disminución de las vibraciones de la torre. Estos resultados representan un buen rango de mejora con respecto al GSPI embebido en FAST.

Finalmente, se ha evaluado el rendimiento global de los controladores comparando la desviación estándar de la potencia de salida generada $\left(\sigma_{P}\right)$, el RMSE del del TTDspFA (RMSs) [19] en el estado estacionario, y el producto de ambas (Tabla 4).

Tabla 4: Comparación de rendimiento

\begin{tabular}{lcll}
\hline Control & $\sigma_{p}$ & RMSs & RMSs. $\sigma_{p}$ \\
\hline FAST & 51.67 & 1 & 0.0705 \\
\hline Hybrid & 28.40 & 0.9930 & 0.0289 \\
\hline
\end{tabular}

Este producto (tercera columna) indica el equilibrio entre ambas métricas, por lo que cuanto menor sea este valor, mejor será el resultado. De nuevo se puede apreciar como la propuesta de control inteligente mejora el controlador de referencia.

\section{CONCLUSIONES}

Las turbinas flotantes son sistemas muy grandes cuyo objetivo es extraer la mayor cantidad de energía posible del viento. Esto hace que su control sea más complejo y además son altamente no lineales y experimentan fuertes cargas externas. En este trabajo se ha abordado el control de cabeceo de un aerogenerador flotante de $5 \mathrm{MW}$ mediante técnicas inteligentes.

Se ha propuesto una estrategia de control híbrida donde se diseña un controlador PD borroso incremental con una función de mapeo difusa, y se aplica al modelo del aerogenerador.

Se han obtenido mejoras relevantes comparando tanto la potencia de salida como las vibraciones del aerogenerador offshore con respecto al controlador de ganancia programada del software de rerencia FAST. En cuanto a las oscilaciones, la estrategia difusa es la que da una respuesta más suave.

Como trabajos futuros se propone mejorar el modelo difuso para incluir reglas específicas para el sistema cuando hay perturbaciones debidas al oleaje. Además, se podría diseñar un sistema difuso que sintonice el controlador PID para implementar la variación de los parámetros en el esquema de ganancia programada.

\section{Agradecimientos}

Este trabajo de investigación ha sido desarrollado con financiación parcial del Proyecto MCI AEI/FEDER RTI2018-094902-B-C21, del Ministerio de Ciencia, Innovación y Universidades.

\section{English summary}

\section{HYBRID PITCH CONTROLLER OPTIMEZED WITH GENETIC ALGORITHM FOR FLOATING WIND TURBINES}

\begin{abstract}
The control of floating wind turbines (FOWT) is complex due to the size of these turbines, the heavy loads they are subjected to and their strongly nonlinear dynamics. Moreover, the control action of the turbine can influence the stability of the floating device. In this work, an intelligent hybrid controller is designed for pitch control of a floating wind turbine. The objective of the control is to reduce the vibration of the floating device without compromising the power output. Specifically, an intelligent fuzzy controller and a fuzzy implementation of the wind speed-pitch angle relationship are developed. The same is validated with the reference provided by the wind turbine simulation software FAST. In addition, the effects of these different control strategies on the vibrations of the structure are analyzed.
\end{abstract}


Keywords: Hybrid system, Pitch control, Fuzzy Control, Vibrations, Floating wind turbine, Wind energy.

\section{REFERENCIAS}

[1] R. M. Aguilar, J. M. Torres, and C. A. Martín, "Aprendizaje automático en la identificación de sistemas. Un caso de estudio en la predicción de la generación eléctrica de un parque eólico," Revista Iberoamericana de Automática e Informática Industrial, vol. 16, no. 1. pp. 114-127, 2019.

[2] I. L. R. Gomes, R. Melício, V. M. F. Mendes, and H. M. I. Pousinho, "Wind power with energy storage arbitrage in day-ahead market by a stochastic MILP approach," $\log . J$. IGPL, vol. 28, no. 4, pp. 570-582, 2020.

[3] M. Mikati, M. Santos, and C. Armenta, "Electric grid dependence on the configuration of a small-scale wind and solar power hybrid system," Renew. energy, vol. 57, pp. 587-593, 2013.

[4] M. Mikati, M. Santos, and C. Armenta, "Modelado y simulación de un sistema conjunto de energía solar y eólica para analizar su dependencia de la red eléctrica," Rev. Iberoam. Automática e Informática Ind., vol. 9, no. 3, pp. 267-281, 2012.

[5] P. M. Rubio, J. F. Quijano, P. Z. López, J. J. F. Lozano, A. G. Cerezo, and J. O. Casanova, "Control inteligente para mejorar el rendimiento de una plataforma semisumergible híbrida con aerogeneradores y convertidores de oleaje: sistema de control borroso para la turbina," Rev. Iberoam. Automática e Informática Ind., vol. 16, no. 4, pp. 480-491, 2019.

[6] C. Serrano-Barreto and M. Santos, "Intelligent Fuzzy Optimized Control for Energy Extraction in Large Wind Turbines," Lect. Notes Comput. Sci. (including Subser. Lect. Notes Artif. Intell. Lect. Notes Bioinformatics), vol. 12490 LNCS, pp. 269276, 2020, doi: 10.1007/978-3-030-623654_26.

[7] C. Kim, E. Muljadi, and C. C. Chung, "Coordinated control of wind turbine and energy storage system for reducing wind power fluctuation," Energies, vol. 11, no. 1, p. 52, 2018.

[8] E. Quiles, E. Garciia, J. Cervera, and J. Vives, "Development of a Test Bench for Wind Turbine Condition Monitoring and Fault Diagnosis," IEEE Lat. Am. Trans., vol. 17, no. 06, pp. 907-913, 2019.

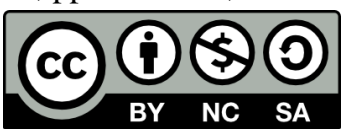

(C) 2021 by the authors. Submitted for possible open access publication under the terms and conditions of the Creative Commons Attribution CC BY-NC-SA 4.0 license (https://creativecommons.org/licenses/byncsa/4.0/deed.es).
[9] J. E. Sierra-Garcia and M. Santos, "Performance Analysis of a Wind Turbine Pitch Neurocontroller with Unsupervised Learning," Complexity, vol. 2020, 2020, doi: 10.1155/2020/4681767.

[10] J. E. Sierra-Garcia, M. Santos, J. E. SierraGarcía, and M. Santos, "Exploring Reward Strategies for Wind Turbine Pitch Control by Reinforcement Learning," Appl. Sci., vol. 10, no. 21, p. 7462, 2020, doi: 10.3390/app10217462.

[11] Z. Civelek, M. Lüy, E. Çam, and H. Mamur, "A new fuzzy logic proportional controller approach applied to individual pitch angle for wind turbine load mitigation," Renewable Energy, vol. 111. pp. 708-717, 2017, doi: 10.1016/j.renene.2017.04.064.

[12] M. Tomás-Rodríguez and M. Santos, "Modelado y control de turbinas eólicas marinas flotantes," Rev. Iberoam. Automática e Informática Ind., vol. 16, no. 4, pp. 381-390, 2019.

[13] L. Acho, "A proportional plus a hysteretic term control design: a throttle experimental emulation to wind turbines pitch control," Energies, vol. 12, no. 10, p. 1961, 2019.

[14] J. Jonkman, S. Butterfield, W. Musial, and G. Scott, "Definition of a 5-MW reference wind turbine for offshore system development," 2009.

[15] M. A. Abdelbaky, X. Liu, and D. Jiang, "Design and implementation of partial offline fuzzy model-predictive pitch controller for large-scale wind-turbines," Renewable Energy, vol. 145. pp. 981-996, 2020, doi: 10.1016/j.renene.2019.05.074.

[16] J.E. Sierra-García, M. Santos. "Lookup Table and Neural Network Hybrid Strategy for Wind Turbine Pitch Control", Sustainability, vol. 13, no. 6, pp. 3235, 2021.

[17] "National Wind Technology Center's Information Portal," NREL.gov. [Online]. Available:

https://www.nrel.gov/wind/nwtc.html.

[18] B. Han, L. Zhou, F. Yang, and Z. Xiang, "Individual pitch controller based on fuzzy logic control for wind turbine load mitigation," IET Renew. Power Gener., vol. 10, no. 5, pp. 687-693, 2016, doi: 10.1049/ietrpg.2015.0320.

[19] C. Zhang and F. Plestan, "Individual/collective blade pitch control of floating wind turbine based on adaptive second order sliding mode," Ocean Eng., vol. 228, no. January, p. 108897, 2021, doi: 10.1016/j.oceaneng.2021.108897. 